\title{
The molecular polar disc in NGC 2768
}

\author{
Alison F. Crocker ${ }^{1}$, Martin Bureau ${ }^{1}$, Lisa M. Young ${ }^{1,2}$, Francoise Combes $^{3}$ \\ ${ }^{1}$ Sub-Department of Astrophysics, University of Oxford, Denys Wilkinson Building, Keble Road, Oxford OX1 3RH \\ ${ }^{2}$ Physics Department, New Mexico Institute of Mining and Technology, Socorro, NM 87801, U.S.A. \\ ${ }^{3}$ Observatoire de Paris, LERMA, 61 Av. de l'Observatoire, 75014, Paris, France
}

\begin{abstract}
We present $\mathrm{CO}(1-0)$ and $\mathrm{CO}(2-1)$ maps of the molecular polar disc in the elliptical galaxy NGC 2768 obtained at the IRAM Plateau de Bure Interferometer. The maps have a resolution of $2{ }^{\prime \prime} 6 \times 22^{\prime \prime} 3$ and $1^{\prime \prime} \cdot 2 \times 1^{\prime \prime} \cdot 2$ for the $\mathrm{CO}(1-0)$ and $\mathrm{CO}(2-1)$ lines, respectively. The $\mathrm{CO}$ maps complete the unique picture of the interstellar medium (ISM) of NGC 2768; the dust, molecular gas, ionised gas and neutral hydrogen (H I) trace the recent acquisition of cold and cool gas over two orders of magnitude in radii (and much more in density). In agreement with the other ISM components, the $\mathrm{CO}$ distribution extends nearly perpendicularly to the photometric major axis of the galaxy. Velocity maps of the $\mathrm{CO}$ show a rotating polar disc or ring in the inner kiloparsec. This cool gas could lead to kinematic substructure formation within NGC 2768. However, the stellar velocity field and $\mathrm{H} \beta$ absorption linestrength maps from the optical integral-field spectrograph SAURON give no indication of a young and dynamically cold stellar population coincident with the molecular polar disc. Very recent or weak star formation, undetectable in linestrengths, nevertheless remains a possibility and could be at the origin of some of the ionised gas observed. Millimetre continuum emission was also detected in NGC 2768, now one of only a few lowluminosity active galactic nuclei with observed millimetre continuum emission.
\end{abstract}

Key words: galaxies: individual: NGC2768 - galaxies: elliptical and lenticular, cD - galaxies: ISM - galaxies: evolution - galaxies: kinematics and dynamics - galaxies: structure

\section{INTRODUCTION}

Galaxy formation scenarios must explain the rather uniform global properties of early-type galaxies as seen in the fundamental plane (e.g. Dressler et al. 1987; Diorgovski \& Davis 1987) while simultaneously accounting for the diversity seen in the internal kinematics and stellar populations (e.g. de Zeeuw et al. 2002). Indeed, the maps of the stellar kinematics of 48 elliptical and S0 galaxies from the SAURON optical integral-field unit (IFU) have shown a wide variety of kinematic substructures, from large embedded discs to small counter-rotating cores (e.g. Emsellem et al. 2004; McDermid et al. 2006). Major mergers have been found to reproduce many of the kinematic substructures observed in the SAURON maps without invoking post-merger star formation (Jesseit et al. 2007). However, many of the kinematically decoupled components (KDCs) in the SAURON maps are very young (Kuntschner et al. 2006; McDermid et al.

\footnotetext{
* Based on observations carried out with the IRAM Plateau de Bure Interferometer. IRAM is supported by INSU/CNRS (France), MPG (Germany) and IGN (Spain).
}

2006), suggesting their stars formed in situ, and not as part of another galaxy.

Over the past twenty years, it has been shown that E/S0s do have detectable amounts of cool and cold gas. Cold dust was first found in the IRAS survey in which around $45 \%$ of ellipticals were detected in both the 60 and $100 \mu \mathrm{m}$ bands (Knapp at al. 1989). Molecular gas detections have often been biased toward dust-rich or infrared-bright galaxies, but two recent surveys do not select on these criteria and give detection rates of $28 \%$ for E/S0s in the SAURON representative sample (Combes, Young \& Bureau 2007) and 78\% for S0s in a volume-limited sample (Sage \& Welch 2006). Particularly interesting for substructure formation are the maps of cold molecular gas provided by interferometric $\mathrm{CO}$ observations (e.g. Young 2002, 2005). These maps often show rotating molecular gas discs on the same spatial scales as KDCs.

While the current evidence indirectly connects molecular gas discs with young KDCs, the details of the connection are still unknown. Do all molecular discs form stellar components? Do stars form everywhere in the molecular disc, or only in the densest regions? Does the regularity of the 
Table 1. Basic properties of NGC 2768. The left and middle columns list the different quantities and their values; the right column lists corresponding references.

\begin{tabular}{llr}
\hline Quantity & Value & Ref. \\
\hline R.A. (J2000.0) & 091137.413 & 1 \\
Dec. (J2000.0) & +600214.86 & 1 \\
Heliocentric Velocity & $1373 \pm 5 \mathrm{~km} \mathrm{~s}^{-1}$ & 2 \\
Distance & $21.5 \mathrm{Mpc}$ & 3 \\
Scale & $1^{\prime \prime}=104 \mathrm{pc}$ & 3 \\
Type (RC3) & $\mathrm{E} 6$ & 4 \\
Type (CAG) & $\mathrm{S} 0$ & 5 \\
Corrected apparent B mag & 10.60 & 6 \\
Corrected absolute B mag & -21.15 & 6 \\
$\mathrm{~L}_{\mathrm{B}}$ & $2.3 \times 10^{10} \mathrm{~L}_{\odot}$ & 7 \\
$\mathrm{~L}_{\mathrm{FIR}}$ & $4.3 \times 10^{8} \mathrm{~L}_{\odot}$ & 7 \\
$\mathrm{~L}_{\mathrm{FIR}} / \mathrm{L}_{\mathrm{B}}$ & $1.9 \times 10^{-2}$ & 7 \\
$\mathrm{~L}_{\mathrm{FIR}} / \mathrm{M}_{\mathrm{H}_{2}}$ & $6.3 \mathrm{~L}_{\odot} / \mathrm{M}_{\odot}$ & 7 \\
\hline
\end{tabular}

References: (1) Nagar et al. (2005); (2) NED; (3) Emsellem et al. (2004); (4) de Vaucouleurs et al. (1991); (5) Sandage \& Bedke (1994); (6) HyperLEDA; (7) Derived quantity using data from NED, IRAS (Moshir et al. 1990), and this paper.

morphology of the molecular disc (presumably indicating how long since the gas was accreted) correlate with the age of the stars? To answer these questions, we must compare the molecular gas morphology and kinematics to the stellar morphology, kinematics and ages in a spatially-resolved manner. This comparison requires interferometric CO maps and optical (or near-infrared) IFU data for many individual galaxies. With this comparison in mind, we have started to obtain CO synthesis maps for the 12 early-type SAURON galaxies recently detected in CO (see Combes et al. 2007, and references therein).

In this paper, we present the first $\mathrm{CO}$ synthesis maps of NGC 2768 and compare the molecular gas to the stellar and ionised gas properties from SAURON. NGC 2768 is classified as an E6 galaxy in RC3 (de Vaucouleurs et al. 1991), an S0 in the Carnegie Atlas of Galaxies (Sandage \& Bedke 1994), and as a fast rotator in the recently proposed kinematic classification scheme of Emsellem et al. (2007). Kim (1989) first discovered both the polar orientation of the inner dust lane and the rotation of the ionized gas about the major axis, suggesting an external accretion origin for the dust and gas. NGC 2768 has a low-luminosity active galactic nucleus (AGN) with a low-ionisation nuclear emission-line region (LINER) spectrum (Heckman 1980), a compact radio core (Nagar. Falcke \& Wilson 2005) and an X-ray source consistent with being a point source (Komossa, Böhringer \& Huchra 1999). General properties of NGC 2768 are listed in Table 1

This paper is organized as follows. In Section 2, we describe the calibration and reduction of the data from the Plateau de Bure Interferometer. Section 3 presents flux measurements for the continuum data and channel maps, total intensity maps and velocity fields for the $\mathrm{CO}$ line data. In Section 4, we discuss the origin and fate of the molecular gas and also consider the contributions to the mm continuum flux from the low-luminosity AGN (LLAGN) and cold dust. We draw our conclusions in Section 5.

\section{OBSERVATIONS AND DATA REDUCTION}

We observed NGC 2768 in December 2005 (C configuration) and August 2006 (D configuration) at the IRAM Plateau de Bure Interferometer (PdBI). The ${ }^{12} \mathrm{CO}(1-0)$ and ${ }^{12} \mathrm{CO}(2-1)$ lines were observed simultaneously using dualband SIS receivers. All six $15 \mathrm{~m}$ antennae were used for the C-configuration observations in December while only five antennae were available for the August observations. Typical system temperatures were $200-300 \mathrm{~K}$ for the $\mathrm{CO}(1-0)$ line in both months and the $\mathrm{CO}(2-1)$ in December. In the worse weather of August, the $\mathrm{CO}(2-1)$ line observations had system temperatures of $300-1000 \mathrm{~K}$. The spectral correlators were centred at $114.743 \mathrm{GHz}$ and $229.482 \mathrm{GHz}$, the transition frequencies of $\mathrm{CO}(1-0)$ and $\mathrm{CO}(2-1)$ corrected for the galaxy's heliocentric velocity of $1373 \mathrm{~km} \mathrm{~s}^{-1}$. The correlator configuration used four $160 \mathrm{MHz}$-wide (417 and $209 \mathrm{~km}$ $\mathrm{s}^{-1}$ for $\mathrm{CO}(1-0)$ and $\mathrm{CO}(2-1)$, respectively) units with a frequency resolution of $1.25 \mathrm{MHz}$ (3.3 and $1.6 \mathrm{~km} \mathrm{~s}^{-1}$ ), covering a total bandwidth of $580 \mathrm{MHz}\left(1670\right.$ and $\left.835 \mathrm{~km} \mathrm{~s}^{-1}\right)$. The correlator was regularly calibrated by a noise source inserted in the IF system.

We obtained visibilities with series of twenty $1 \mathrm{~min}$ integrations on source, followed by three $45 \mathrm{~s}$ phase and amplitude calibrations on a nearby quasar, 0836+710. As 0836+710 was not bright enough, we used 3C84 (25 December), 0528+134 (25 August) and MWC349 (28 August) as flux calibrators. Comparing the flux measured for $0836+710$ on 28 August to that measured on 25 December suggests an uncertainty in our flux calibration of $\approx 20 \%$. This is unfortunately not a very good estimate as the true flux of $0836+710$ may have varied over the 8 month interval.

The data were reduced with the Grenoble Image and Line Data Analysis System (GILDAS) software packages CLIC and MAPPING (Guilloteau \& Lucas 2000). Using CLIC, we first calibrated the data using the standard pipeline. Data obtained during periods of bad weather were flagged and then ignored. After calibration, we used MAPPING to create two data cubes for both lines with velocity planes separated by $40 \mathrm{~km} \mathrm{~s}^{-1}$ and $60 \mathrm{~km} \mathrm{~s}^{-1}$. The primary beam size is $44^{\prime \prime}$ for the $\mathrm{CO}(1-0)$ observations and $22^{\prime \prime}$ for the $\mathrm{CO}(2-1)$ observations. We choose the spatial dimensions of the datacube to be twice the diameter of the primary beam, $88^{\prime \prime} \times 88^{\prime \prime}$ and $44^{\prime \prime} \times 44^{\prime \prime}$ for the $\mathrm{CO}(1-0)$ and $\mathrm{C}(2-1)$ lines, respectively. The synthesized beam sizes are $2^{\prime \prime} .6 \times 2$.' 3 for $\mathrm{CO}(1-0)$ and $1^{\prime \prime} .2 \times 1^{\prime \prime} .2$ for $\mathrm{CO}(2-1)$. We thus choose spatial pixels of $.7^{\prime \prime} \times .7^{\prime \prime}$ and $.35^{\prime \prime} \times .35^{\prime \prime}$ for the $\mathrm{CO}(1-0)$ and $\mathrm{CO}(2$ 1) lines, respectively. As we detected continuum emission, we subtracted the continuum using the task contsub in the Multichannel Image Reconstruction, Image Analysis and Display (MIRIAD) software package (Sault, Teuben \& Wright 1995). The dirty beam has small side lobes that necessitated cleaning the datacube. The cleaning was done using the Högbom method (Högbom 1974); we stopped cleaning in each velocity plane after the brightest residual pixel had a value lower than the rms of the uncleaned datacube.

To constrain the continuum emission, we selected channels at least $40 \mathrm{~km} \mathrm{~s}^{-1}$ away from the highest and lowest velocity channels with any line emission in the cleaned data cubes. The very edges of the $580 \mathrm{MHz}$ bandwidth were also avoided. The resulting continuum windows were 356 and $200 \mathrm{MHz}$ wide at 115 and $230 \mathrm{GHz}$, respectively. We used 


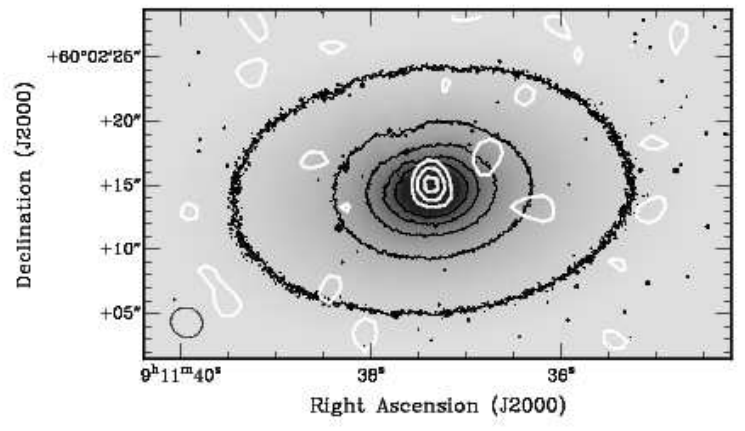

Figure 1. $3 \mathrm{~mm}$ continuum contours (white) over a Hubble Space Telescope (HST) Advanced Camera for Surveys (ACS) image (F814W filter, black contours) of NGC 2768. Contours are at 2, 4 , and $6 \sigma$, where $\sigma=0.28 \mathrm{mJy}^{\text {beam }}{ }^{-1}$. The synthesized beam is plotted in the lower left corner.

the MAPPING tasks with the same parameters as for the line data to create clean images of the continuum at both frequencies. We fit point-source models in the $u v$ plane to find the flux of the $3 \mathrm{~mm}$ continuum and an upper limit for the $1 \mathrm{~mm}$ continuum. The continuum emission is spatially unresolved at both frequencies.

\section{RESULTS}

We detected extended emission in both the $\mathrm{CO}(1-0)$ and $\mathrm{CO}(2-1)$ lines and point-source continuum emission at $3 \mathrm{~mm}$.

\subsection{Continuum emission}

The continuum maps at 1 and $3 \mathrm{~mm}$ both show peaks at the centre of NGC 2768. The $3 \mathrm{~mm}$ continuum map has a peak of over $6 \sigma$ consistent with being a point source (see Fig. 1). Fitting a point-source model in the $u v$-plane yields a flux density of $1.94 \mathrm{mJy}$ at a right ascension of 0911 37.38 and declination of +600214.8 . This position is off the VLA coordinates of Nagar et al. (2005) by $-0.5^{\prime \prime}$ in right ascension and $-0.1^{\prime \prime}$ in declination. Given the $2^{\prime \prime} .6 \times 2$.' 3 beam, this source is however still identifiable with the radio source, despite the large offset in right ascension. The $1 \mathrm{~mm}$ continuum map shows a weak peak at the same location. Fitting a point-source model in the $u v$-plane to the same coordinates as found for the $3 \mathrm{~mm}$ continuum emission gives a flux density of $1.56 \mathrm{mJy}$ with a rms noise $\sigma=0.75 \mathrm{mJy}$. We thus do not consider this a reliable determination of the flux density at $1 \mathrm{~mm}$, and instead adopt a $3 \sigma$ upper limit of $2.25 \mathrm{mJy}$.

\subsection{CO line emission}

Fig. 2 shows the channel maps for the $\mathrm{CO}(1-0)$ and $\mathrm{CO}(2-$ 1) lines from the $60 \mathrm{~km} \mathrm{~s}^{-1}$ datacubes. To draw attention to the overall morphology and kinematics of the $\mathrm{CO}$, we created integrated intensity maps, mean velocity field maps and position-velocity (PV) diagrams using the $40 \mathrm{~km} \mathrm{~s}^{-1}$ datacubes, for better velocity resolution (Figs. 3 and 4). To make these maps, we first created a smoothed cube by smoothing with a 2D gaussian (FWHM of 4 pixels) spatially and hanning smoothing by 3 channels in velocity. We then computed the moments by integrating in velocity over the pixels in the original cube that corresponded to pixels above $3 \sigma$ in the smoothed cube. As the emission is oriented primarily north-south, we created the PV diagrams by computing the zeroth moment of the masked cube in the Declinationvelocity plane. This smoothing-masking procedure excludes much, but not all, noise from the moment maps.

As shown in the integrated intensity maps of both transitions, the CO is distributed somewhat asymmetrically, although with the signal just above $3 \sigma$ in most of the velocity channels, we have to be wary of the contribution of noise even with the smoothing and masking procedure applied. The smaller $\mathrm{CO}(2-1)$ beam resolves two peaks in the $\mathrm{CO}$ intensity, one directly south of the galaxy centre and one to the north by north-east. The two clear $\mathrm{CO}(2-1)$ peaks suggest that the densest gas might be in a disc or ring seen nearly edge-on. The lower-resolution $\mathrm{CO}(1-0)$ data blend these two separate peaks into one peak elongated in the direction of the $\mathrm{CO}(2-1)$ peaks. In addition, the $\mathrm{CO}(1-0)$ data pick up an extended structure along $\mathrm{PA} \approx 30^{\circ}$, especially prominent to the north-east. While this feature is absent in the $\mathrm{CO}(2-1)$ maps, it appears in both the 40 and $80 \mathrm{~km} \mathrm{~s}^{-1}$ channels of the $\mathrm{CO}(1-0)$ data, suggesting it is not just noise. The $\mathrm{CO}(2-$ 1) observations must miss this molecular gas through some combination of the more limited primary beam size, noise, and a potentially lower excitation temperature of the infalling gas. Overall, the molecular gas distribution suggests that we are observing the formation of an inner polar ring or disc, with gas in the outskirts of the inner kiloparsec still being accreted onto the settled structure.

The velocity maps (Fig. 3) and PV diagrams (Fig. 4) support this interpretation. The overlay of the $\mathrm{CO}(1-0) \mathrm{PV}$ contours on the $\mathrm{CO}(2-1) \mathrm{PV}$ pixel map in Fig. 4 shows that the rotation of the main body of molecular gas is in good agreement for the two lines. Unfortunately, the quality of the data does not allow us to reliably determine whether the molecular gas is in a ring or a disc. For simplicity and given the hint of a flattening of the rotation at offsets larger than $\approx 22^{\prime \prime}$, we refer to it as a disc for the remainder of the paper but refrain from making any claims that depend on whether it is a disc or a ring. The polar rotation is consistent with the velocity field of the more extended ionized gas (Sarzi et al. 2006). However, the CO(1-0) mean velocity map is irregular in the northern part of the galaxy. This irregularity is caused by the lower velocity (40 and $80 \mathrm{~km} \mathrm{~s}^{-1}$ ) molecular gas making up the extension to the north-east of the galaxy centre, as can be clearly seen in the $\mathrm{CO}(1-0) \mathrm{PV}$ contours. The velocity and extent of this gas lead to the conclusion that it must not yet be part of the central regularly rotating structure, but instead be in the process of accreting onto this structure. While other minor irregular features in the velocity maps may indicate further irregularities in the molecular gas kinematics, the accidental inclusion of a noise peak from a particular channel map could influence the velocity map in the same way, thus we hesitate to analyse these features.

To construct integrated spectra and determine total fluxes, we selected the pixels in the central non-zero parts of the two integrated intensity maps as our spatial regions of interest. Integrating the original datacubes over these spa- 

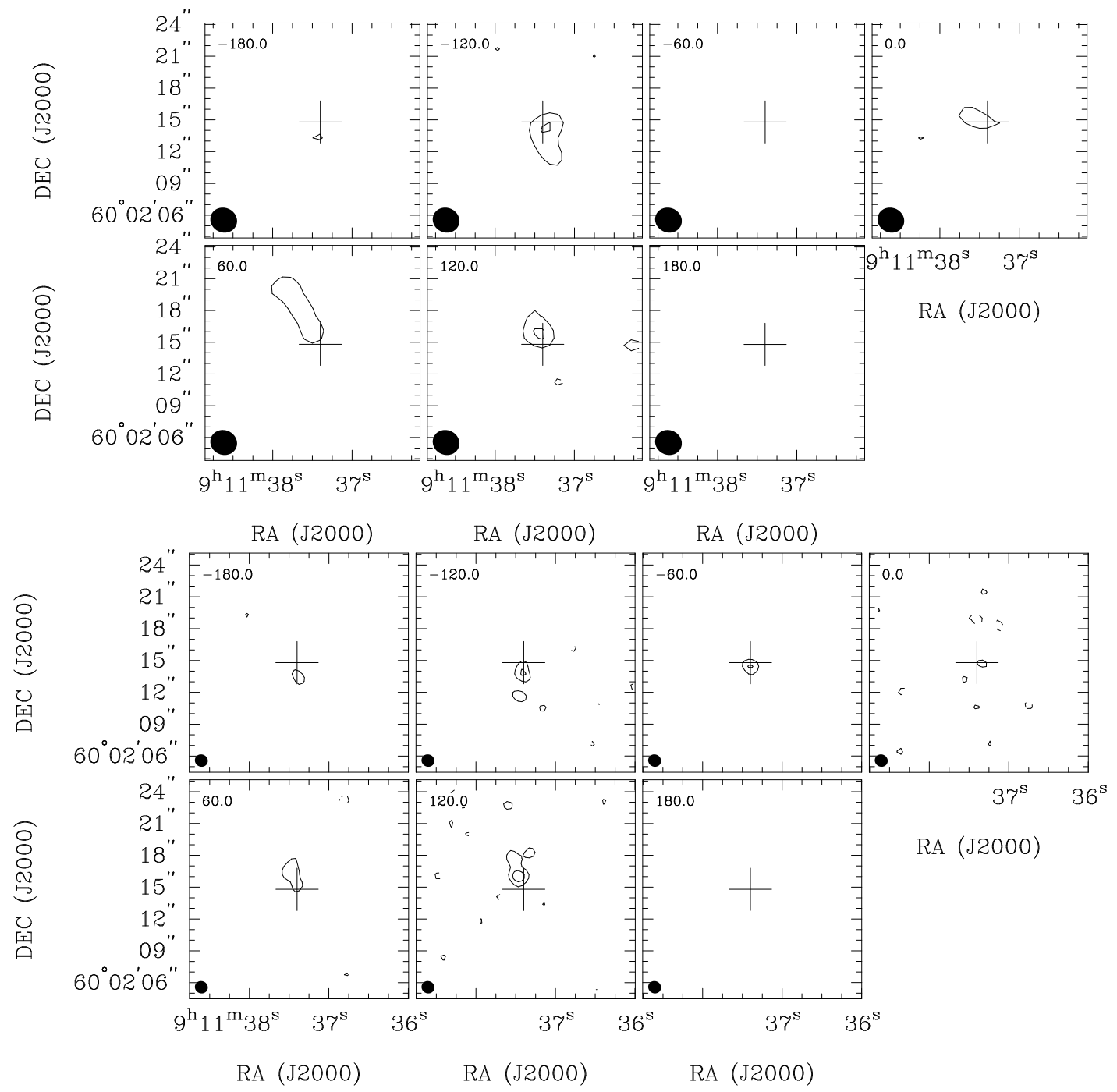

Figure 2. Channel maps of NGC 2768. Top: ${ }^{12} \mathrm{CO}(1-0)$ line. Bottom: ${ }^{12} \mathrm{CO}(2-1)$ line. The channels are $60 \mathrm{~km} \mathrm{~s}{ }^{-1}$ wide and contours are plotted at -3 (dashed), 3, 6 , and $9 \sigma$ with $\sigma=1.0$ mJy beam $^{-1}$ for $\mathrm{CO}(1-0)$ and $\sigma=1.6 \mathrm{mJy}^{-1}$ beam $^{-1}$ for $\mathrm{CO}(2-1)$. The number in the top left corner of each frame is the central velocity of that frame, relative to the observed central velocity of $V_{\text {sys }}=1373 \mathrm{~km} \mathrm{~s}{ }^{-1}$. The cleaned beam is shown in the bottom left corner of each frame. The cross marks the centre of the $5 \mathrm{GHz}$ radio continuum (Nagar et al. 2005).

tial regions gave us the spectra shown in Fig. 5. Spectra from the $30 \mathrm{~m}$ single-dish observations of Combes et al. (2007) are over-plotted in dotted lines.

Comparison with the Combes et al. (2007) 30m data shows that the double-peaked profile seen in both lines in the interferometric data is absent in the $30 \mathrm{~m}$ single-dish data. While the large amount of noise in the $30 \mathrm{~m}$ spectra certainly contributes to the difference in the spectral profiles, we also note that both $30 \mathrm{~m}$ spectra are biased toward the positive relative velocities seen on the northern side of the galaxy. If the 30m's pointing were off to the north, it would explain why intensity is preferentially missing at the negative velocities. However, the intensities at positive relative velocities at $1 \mathrm{~mm}$ are also much lower than found with the interferometer. This difference suggests that the pointing may have also been off in the east-west direction. The $1 \mathrm{~mm}$ spectrum suffered much more from this pointing error as the half-
Table 2. CO fluxes and molecular mass estimates in NGC 2768.

\begin{tabular}{lccc}
\hline Line & Instrument & $\begin{array}{c}\text { Flux } \\
\left(\mathrm{Jy} \mathrm{km} \mathrm{s}^{-1}\right)\end{array}$ & $\begin{array}{c}\mathrm{M}_{\mathrm{H}_{2}} \\
\left(10^{7} \mathrm{M}_{\odot}\right)\end{array}$ \\
\hline${ }^{12} \mathrm{CO}(1-0)$ & $30 \mathrm{~m}$ & $10.4 \pm 1.6 \pm 1.0$ & $6.4 \pm 1.0 \pm 0.6$ \\
${ }^{12} \mathrm{CO}(1-0)$ & $\mathrm{PdB}$ & $11.3 \pm 1.3 \pm 2.3$ & $6.8 \pm 0.8 \pm 1.4$ \\
${ }^{12} \mathrm{CO}(2-1)$ & $30 \mathrm{~m}$ & $18.6 \pm 2.5 \pm 1.9$ & \\
${ }^{12} \mathrm{CO}(2-1)$ & $\mathrm{PdB}$ & $30.6 \pm 1.7 \pm 6.1$ & \\
\hline
\end{tabular}

Errors reported are random and systematic (flux calibration) errors, respectively.

power beam-width is only $11^{\prime \prime}$. We note that errors in the flux calibrations could also explain some of the discrepancy.

To estimate the total $\mathrm{CO}$ flux in each line, we integrated the spectra over the velocity range with observed emission, i.e. from 1193 to $1553 \mathrm{~km} \mathrm{~s}^{-1}$. Table 2 reports the calculated total fluxes, $11.3 \mathrm{Jy} \mathrm{km} \mathrm{s}^{-1}$ at $\mathrm{CO}(1-0)$ and 

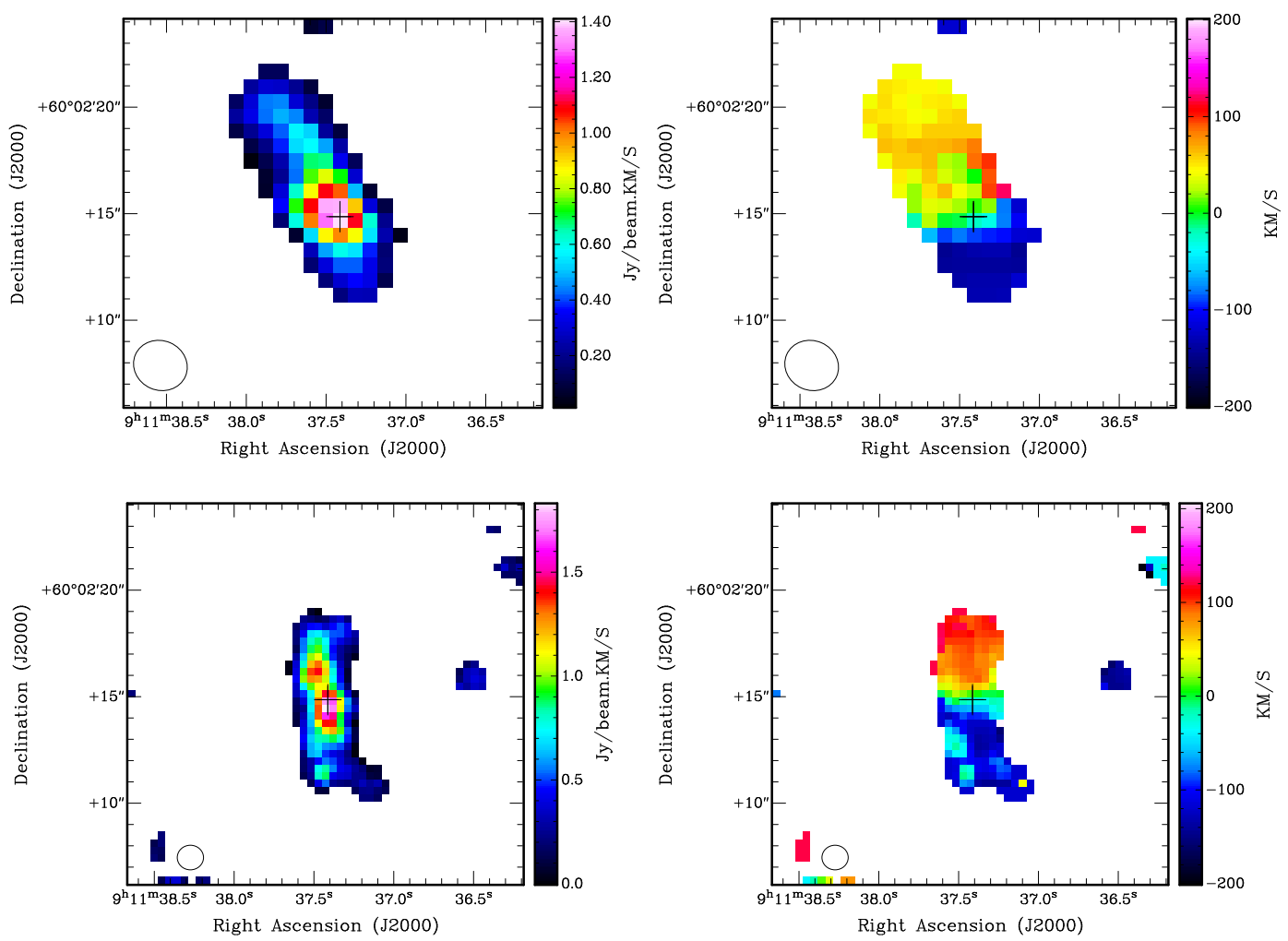

Figure 3. Left: CO integrated intensity maps of NGC 2768. Right: CO mean velocity maps. Top: $\mathrm{CO}(1-0)$. Bottom: CO(2-1). The synthesized beam is shown in the bottom-left corner of each map. The black cross marks the centre of the $5 \mathrm{GHz}$ radio continuum (Nagar et al. 2005). White pixels have been masked according to the procedure described in Section 3.2.

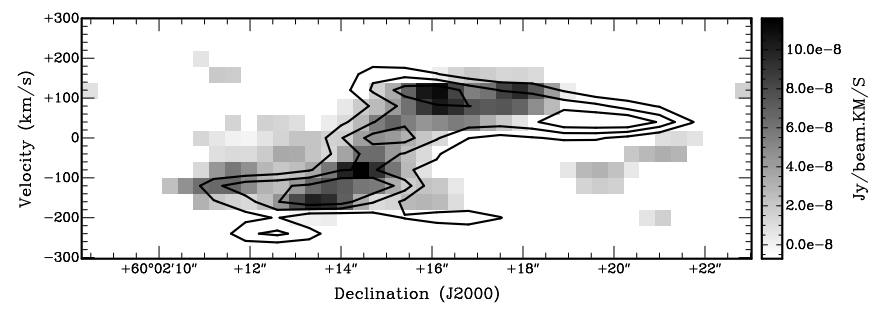

Figure 4. Position-velocity diagram of NGC 2768 along the polar axis. The pixels show the $\mathrm{CO}(2-1)$ emission, while the contours show the $\mathrm{CO}(1-0)$ emission at 3,6 , and $9 \times 10^{8} \mathrm{Jy}_{\text {beam }}^{-1} \mathrm{~km}$ $\mathrm{s}^{-1}$. White pixels have been masked according to the procedure described in Section 3.2.

$30.6 \mathrm{Jy} \mathrm{km} \mathrm{s}^{-1}$ at $\mathrm{CO}(2-1)$. Fluxes for the $30 \mathrm{~m}$ observations of Combes et al. (2007) are also reported; these fluxes have been calculated over identical velocity ranges as their interferometric counterparts. For $\mathrm{CO}(1-0)$, the fluxes agree within the noise and the errors in the flux calibration. For the $\mathrm{CO}(2-1)$ line, the $30 \mathrm{~m}$ misses much of the flux seen by the interferometer, especially at negative relative velocities where the flux from the $30 \mathrm{~m}$ is $8.2 \mathrm{Jy} \mathrm{km} \mathrm{s}^{-1}$ less than for the interferometer. As we have noted above, we believe much of this difference to be due to a pointing error.

We used the total fluxes in $\mathrm{CO}(1-0)$ to compute the to- tal molecular hydrogen mass using the formula $M\left(\mathrm{H}_{2}\right)=$ $\left(1.22 \times 10^{4} \mathrm{M}_{\odot}\right) D^{2} * S_{\mathrm{CO}}$, where $\mathrm{D}$ is the distance measured in $\mathrm{Mpc}$ and $S_{\mathrm{CO}}$ is the total $\mathrm{CO}(1-0)$ flux. This formula comes from using the standard $\mathrm{CO}$ to $\mathrm{H}_{2}$ conversion ratio $N\left(\mathrm{H}_{2}\right) / I(\mathrm{CO})=3 \times 10^{20} \mathrm{~cm}^{-2}$, where $N\left(\mathrm{H}_{2}\right)$ is the column density of $\mathrm{H}_{2}$ and $I(\mathrm{CO})$ is the $\mathrm{CO}(1-0)$ intensity in $\mathrm{K} \mathrm{km} \mathrm{s}^{-1}$. The total $\mathrm{H}_{2}$ mass detected by our interferometric observations is then $6.8 \times 10^{7} \mathrm{M}_{\odot}$. As nearly all the $\mathrm{CO}(1-0)$ flux comes from the nascent polar disc seen in the integrated intensity maps, this gives a rough estimate of the molecular mass already in the central polar disc. We estimate the integrated line ratio $R_{21}=\mathrm{CO}(2-1) / \mathrm{CO}(1-0) \approx 0.7$, indicating optically thick molecular gas that is subthermally excited, as expected for a non-starburst galaxy. This ratio is computed assuming we have mapped the full extent of the $\mathrm{CO}$ in both lines in our interferometric maps. As the $\mathrm{CO}$ distribution in both lines is only about half the diameter of the HPBW of the primary beam, this assumption is reasonable.

\section{DISCUSSION}

\subsection{Origin of the Molecular Gas}

With H I data from Morganti et al. (2006), dust maps from HST images (e.g. Fig. 6), and the molecular gas maps of section 3 , we now have a fairly complete census of the cool ISM in and around NGC 2768. The most striking feature, besides the fact that the ISM lies in the polar plane, is the 

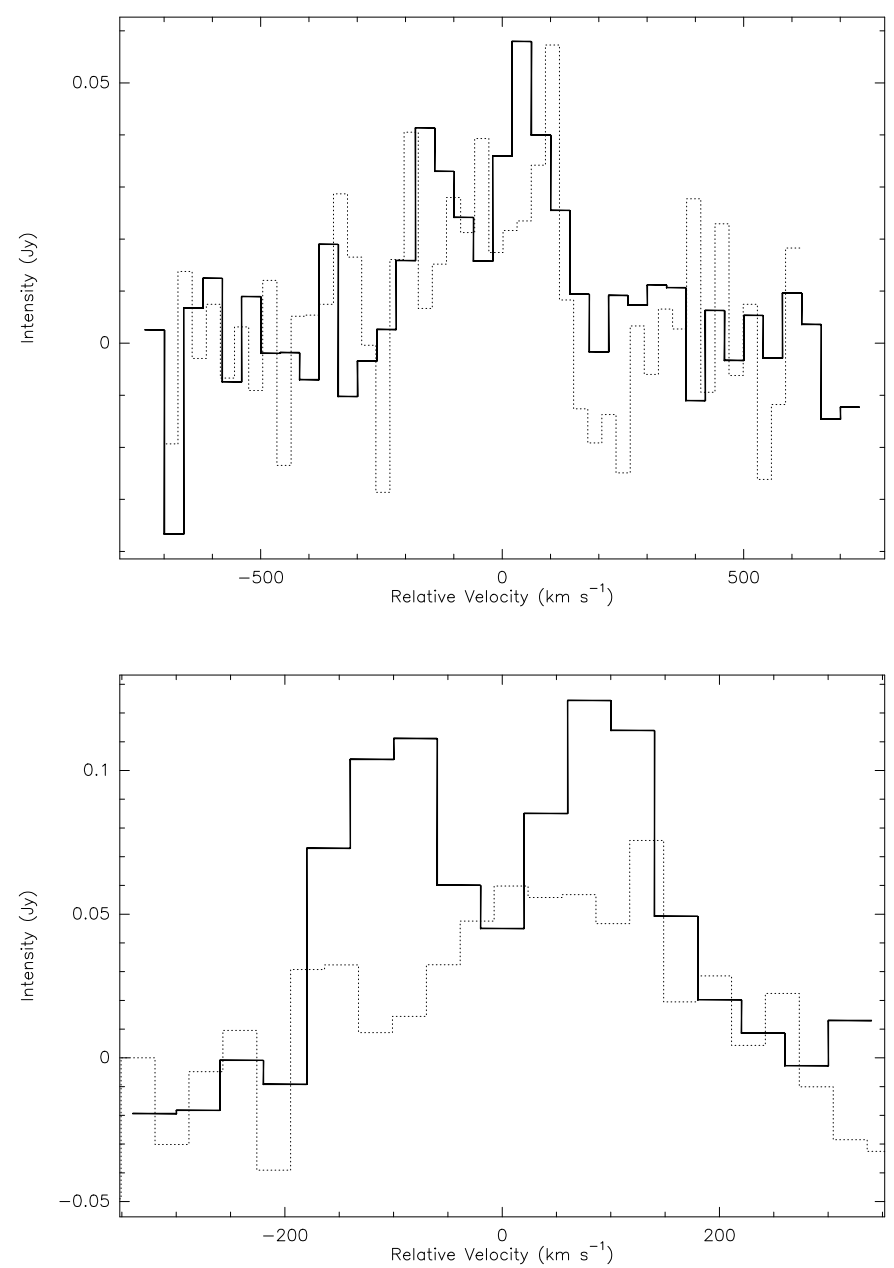

Figure 5. Solid lines show integrated spectra of the $\mathrm{CO}(1-0)$ line (top) and $\mathrm{CO}(2-1)$ line (bottom) in NGC 2768. The dotted lines show the single-dish spectra of Combes et al. (2007).

clear link between all components. All observed phases of the cool ISM extend to the north-east, first in $\mathrm{CO}$, then dust, then H I (Fig. 6). This shared asymmetry ranges from the inner kiloparsec in the molecular gas to over $30 \mathrm{kpc}$ in H I. This link between the different ISM components clearly indicates that they were all accreted from the same source. It also suggests that the gas accretion will continue for some time, perhaps in discrete episodes.

This extended and asymmetric morphology along with the misaligned kinematics of the cool gas also firmly indicates an external origin. We consider three options: a minor merger with a gas-rich dwarf, tidal accretion from a gas-rich galaxy, and accretion from an $\mathrm{HI}$ filament. The regularity of both the photometry and stellar kinematics of NGC 2768 argues against the recent merger of a gas-rich dwarf galaxy. No stellar tidal tail nor other irregularity has been observed, although the data we have access to do not pose particularly stringent upper limits (i.e. the optical photometry is relatively shallow). The accretion of primordial (metal-free) H I scenario fails to explain both the dust and $\mathrm{CO}$ observed. We thus consider tidal accretion as the most likely scenario.

The loose-group centred on NGC 2768, catalogued as Lyon Group of Galaxies (LGG) 167 (Garcia 1993), contains many potential gas-donor galaxies (see Table 3). The H I datacube obtained by Morganti et al. (2006) reveals neutral hydrogen around 4 lower-luminosity galaxies within a 150 $\mathrm{kpc}$ projected separation from NGC 2768. The H I velocities indicate group membership for all of these galaxies. Considering the wide range $(0.1$ to 6$)$ of $\mathrm{H}$ I mass to blue luminosity ratio $\left(M_{\mathrm{H} I} / L_{B}\right)$ values found in late-type dwarf galaxies (Swaters 1999), any of these galaxies could have provided the $\sim 10^{8} \mathrm{M}_{\odot}$ of $\mathrm{H}$ I found around NGC 2768 without requiring an unreasonable amount of $\mathrm{HI}$ for its blue luminosity.

Including all galaxies within $400 \mathrm{kpc}$ in projected separation and $400 \mathrm{~km} \mathrm{~s}^{-1}$ in relative line-of-sight velocity from NGC 2768, we created a list of known galaxies that may have recently interacted with NGC 2768 (Table 3). Given the relaxed appearance of the ionised gas in the SAURON map (Sarzi et al. 2006), we estimate that this gas must have been present for at least 3-10 dynamical timescales, or 0.20.7 Gyr. Assuming $\sigma_{\text {group }}=200 \mathrm{~km} \mathrm{~s}^{-1}$ as a reasonable three-dimensional velocity dispersion for a small group, we estimated the time since each galaxy could have interacted with NGC 2768,

$\Delta t=\frac{\Delta R}{\sqrt{\sigma_{\text {group }}^{2}-\Delta V_{\mathrm{sys}}^{2}}}$,

where $\Delta R$ is the projected separation and $\Delta V_{\text {sys }}$ the line-ofsight velocity relative to NGC 2768. Values of this estimated interaction time are listed in Table 3 This analysis shows that the nearby Scd galaxy UGC 4808 is most likely to have interacted with NGC 2768 recently and provided the cool ISM. UGC 4808 can be seen in the bottom right corner of Fig. 6] Its alignment with the large-scale H I supports this conclusion.

\subsection{Fate of the Molecular Gas: Star Formation?}

In NGC 2768, we observe no definitive signs of young stars as seen in the other five SAURON early-type galaxies with mapped CO emission (NGC 2865, Schinnerer \& Scoville 2002; NGC 3032, NGC 4150, NGC 4459 and NGC 4526, Young. Bureau \& Cappellari 2008). Excluding NGC 2685, in which the CO lies outside of the SAURON field-ofview, young stars in these galaxies are traced by high $\mathrm{H} \beta$ linestrengths (all), cold stellar components that share the $\mathrm{CO}$ kinematics (all except NGC 3032), and low [O III] to $\mathrm{H} \beta$ emission line ratios (all except NGC 4150).

The $\mathrm{H} \beta$ linestrength index of NGC 2768 is $1.70 \AA$ within a central $9^{\prime \prime}$ radius aperture (one eighth of the effective radius) and remains near this level over the entire SAURON field (Kuntschner et al. 2006; Fig. 7). Higher resolution but lower signal-to-noise data from the OASIS integral-field spectrograph give slightly larger values for the $\mathrm{H} \beta$ linestrength (McDermid et al. 2006). However, the discrepancy between the OASIS and SAURON values is probably attributable to the difficulty of subtracting the strong $\mathrm{H} \beta$ emission before fitting the linestrengths, and the higher signal-to-noise data from SAURON provide a better estimate. Combining line-strength indices $(\mathrm{H} \beta$, Fe 5015 , and $\mathrm{Mg}$ b) with stellar population models, Kuntschner et al. (in preparation) show that NGC 2768 has a fairly uniform, old stellar population. However, linestrengths are not sensitive to very recent star formation nor to a very small percentage 
Table 3. Basic properties of galaxies near NGC 2768. This table lists all galaxies with a projected distance differing by less than 400 kpc and a redshift differing by less than $400 \mathrm{~km} \mathrm{~s}^{-1}$ from NGC 2768. Only galaxies with known redshifts are included.

\begin{tabular}{lrrrrrrrr}
\hline Name & $\Delta \theta$ & $\begin{array}{r}\Delta \mathrm{R} \\
(\mathrm{kpc}) \\
(1)\end{array}$ & $\begin{array}{r}\Delta \mathrm{V}_{\mathrm{sys}} \\
\left(\mathrm{km} \mathrm{s}^{-1}\right)\end{array}$ & $\begin{array}{r}B_{\mathrm{T}} \\
(\mathrm{mag}) \\
(4)\end{array}$ & $\begin{array}{r}M_{\mathrm{H} \mathrm{I}} \\
\left(10^{8} \mathrm{M}_{\odot}\right)\end{array}$ & $\begin{array}{r}M_{\mathrm{H} \mathrm{I}} / L_{B} \\
\left(\mathrm{M}_{\odot} / \mathrm{L}_{\odot}\right)\end{array}$ & $\begin{array}{r}\Delta t \\
(\mathrm{Gyr}) \\
(8)\end{array}$ & $\begin{array}{r}\text { Type } \\
(\mathrm{NED}) \\
(9)\end{array}$ \\
\hline NGC 2768 & - & - & - & 10.60 & 1.7 & 0.007 & - & $\mathrm{E}$ \\
UGC 04808 & $11 ! 5$ & 72 & -78 & 15.03 & 1.6 & 0.41 & 0.38 & $\mathrm{Scd}$ \\
PGC 025982 & $16 ! 5$ & 103 & -236 & 14.71 & & & & \\
MAILYAN 038 & $19 ! 8$ & 124 & 187 & & 0.3 & & 1.71 & \\
PGC 2599651 & $21 ! 8$ & 136 & 197 & 17.05 & 0.4 & 0.63 & 3.83 & \\
PGC 2601563 & $22 ! 5$ & 141 & -325 & 17.36 & 0.6 & 1.4 & & \\
SDSS J091531.98+594948.5 & $31 ! 9$ & 200 & -5 & 17.14 & & & 0.98 & $\mathrm{SA}(\mathrm{s}) \mathrm{c}$ \\
NGC 2742 & $40 ! 2$ & 252 & -84 & 11.38 & 18.4 & 0.16 & 1.36 & $\mathrm{~S}$ \\
NGC 2726 & $50 ! 6$ & 317 & 145 & 12.56 & 1.8 & 0.05 & 2.25 & $\mathrm{Sa} ?$ \\
\hline
\end{tabular}

(1) Galaxy name. (2) Angular separation. (3) Projected separation (assuming a distance of 21.5 Mpc). (4) Relative line-of-sight velocity. (5) Total corrected apparent blue magnitude (HyperLEDA). (6) Mass of neutral hydrogen (Morganti et al. 2006, except for

NGC 2742 - Broeils \& van Woerden 1994 and NGC 2726 - Haynes et al. 1988). (7) H I mass to total blue luminosity ratio. (8) Estimate for time since a possible interaction with NGC 2768. (9) Galaxy classification (NED).

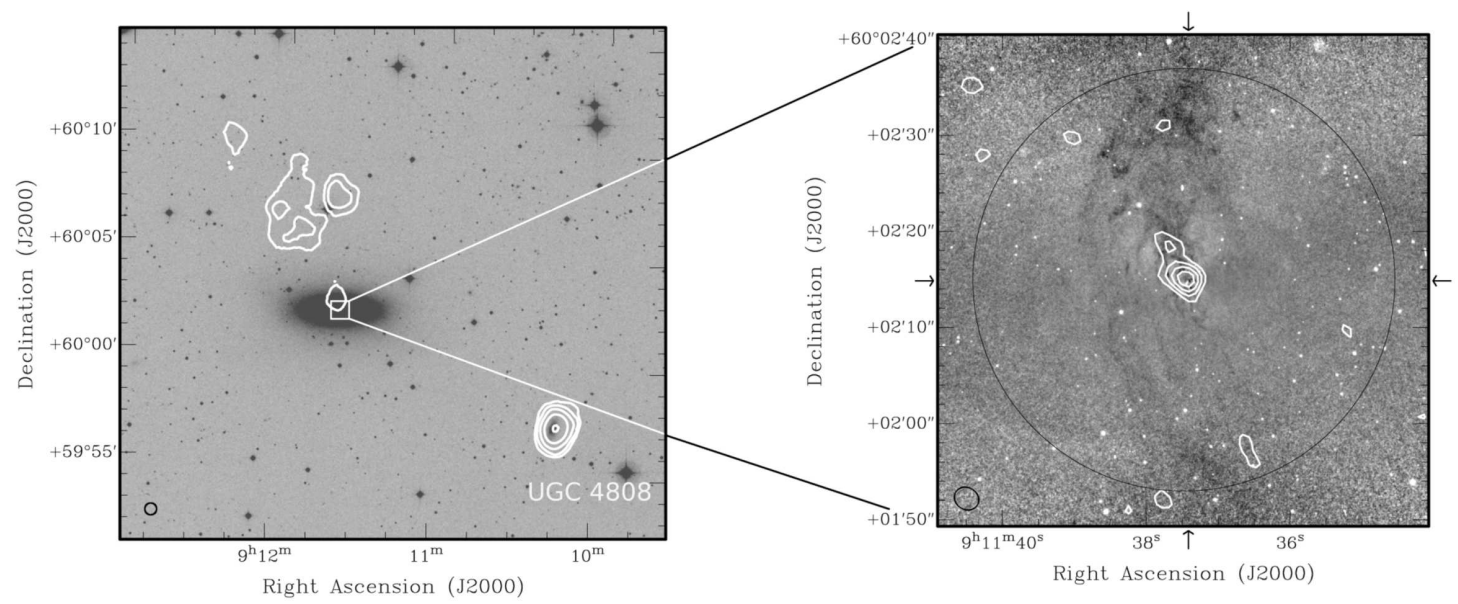

Figure 6. The cool ISM in NGC 2768. The left panel shows the H I surface density distribution in contours of 2, 4, 8, 16 and $32 \times 10^{19}$ atom $\mathrm{cm}^{-2}$ plotted over an R-band Digital Sky Survey (DSS) image. UGC 4808 is visible in the bottom right-hand corner. The right panel shows the $\mathrm{CO}(1-0)$ intensity distribution in contours of $0.5,0.75,1.0$ and $1.25 \mathrm{Jy} \mathrm{km} \mathrm{s}^{-1}$ plotted over a dust map of NGC 2768 obtained by dividing the HST F814W ACS image by an ellipse model fit. In the right panel, the outer circle indicates the $44^{\prime \prime}$ HPBW of the $\mathrm{CO}(1-0)$ primary beam of the interferometer and the arrows indicate the galaxy centre. The black ellipses in the bottom left corners of both panels show the synthesized beams of the H I and CO observations, respectively.

of young stars superposed on a dominant older stellar population, so linestrengths cannot rule out particularly recent and/or weak star formation.

The SAURON map of the stellar velocity dispersion shows a dramatic drop of $\approx 45 \mathrm{~km} \mathrm{~s}^{-1}$ in the innermost $8^{\prime \prime}$ (Emsellem et al. 2004; Fig. 7). This drop in dispersion is also clearly seen in long-slit data (Simien \& Prugniel 1997) and in OASIS data (McDermid et al. 2006). Young stars born from dynamically cold gas in a cicumnuclear disc can produce such a drop in dispersion (Emsellem et al. 2001; Wozniak et al. 2003), but they should also produce a noticeable effect in the velocity map. However, the velocity field of
NGC 2768 lacks any signature of a dynamically cold stellar disc or ring. Thus the stellar velocity dispersion drop cannot be directly attributed to the formation of a stellar component either from the molecular polar ring itself, or from gas settled into the main plane of the galaxy as may occur during polar accretion events (Bournaud \& Combes 2003). Additionally, it is difficult to associate this drop to a young stellar population, as no coincident increase in $\mathrm{H} \beta$ linestrength is observed. We conclude that no kinematic component visible in the SAURON maps is directly related to the molecular gas.

Particularly low ratios of $[\mathrm{O}$ III $]$ to $\mathrm{H} \beta[\log ([\mathrm{O}$ III $] / \mathrm{H} \beta)$ 

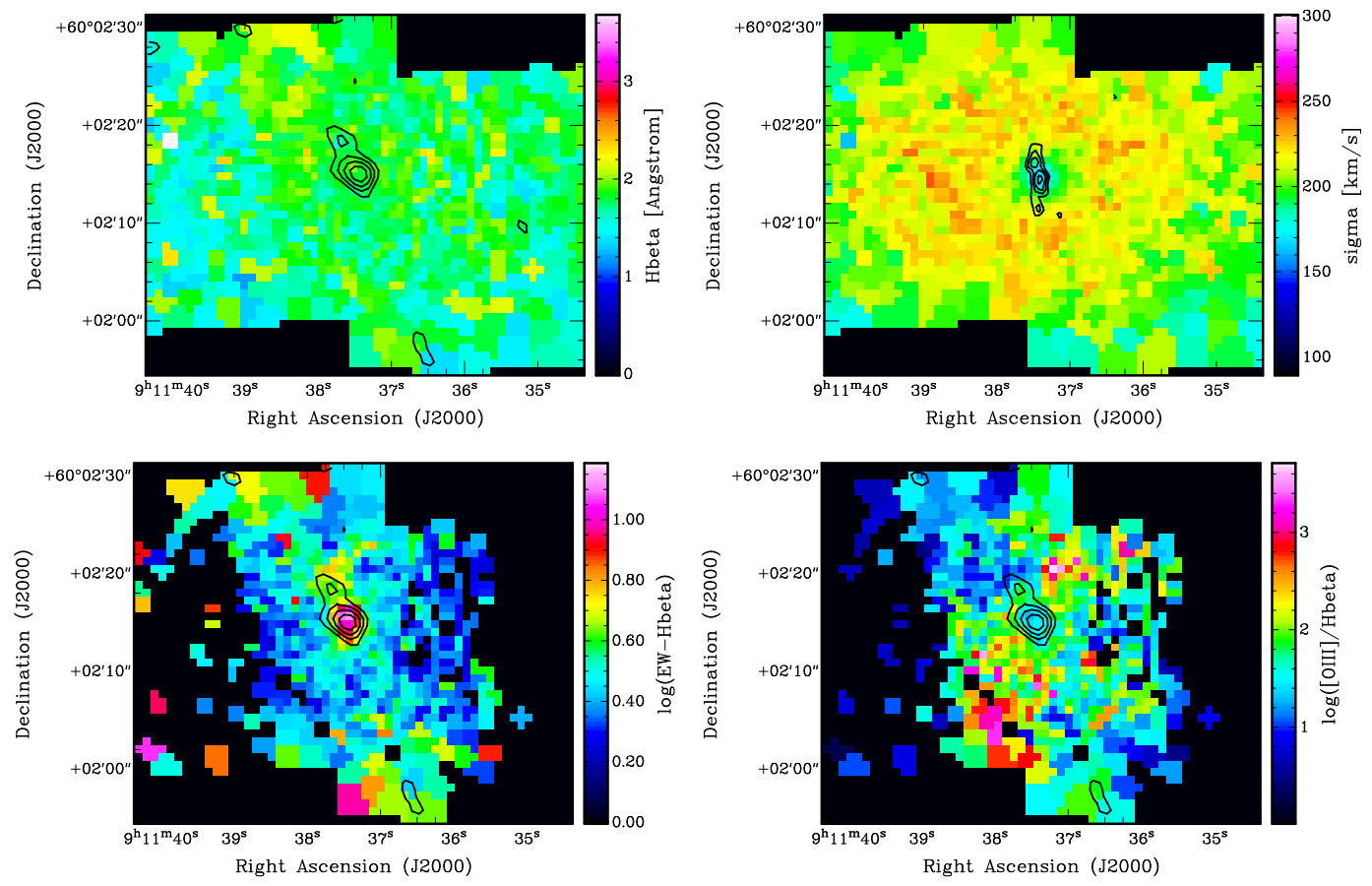

Figure 7. Top Left: $\mathrm{CO}(1-0)$ contours of NGC 2768 over the SAURON H $\beta$ absorption linestrength map (Kuntschner et al. 2006). No significant change in linestrength is coincident with the molecular gas. Top Right: $\mathrm{CO}(2-1)$ contours over the stellar velocity dispersion map (Emsellem et al. 2004). The CO is co-spatial with the dispersion drop. Bottom Left: CO(1-0) contours over a map of the equivalent width of $\mathrm{H} \beta$ emission (Sarzi et al. 2006). Note the increase in equivalent width near the extended CO peak to the north-east. Bottom Right: $\mathrm{CO}(1-0)$ contours over a map of the flux ratio of [O III] to $\mathrm{H} \beta$ emission (Sarzi et al. 2006).

$<-0.2]$ indicate star formation as the source of ionisation (Ho et al. 1997). In NGC 2768, $\log ([\mathrm{O}$ III $] / \mathrm{H} \beta)$ ranges from about 0.0 to 0.5 . In this range, the ionisation can come from sources other than star formation. The four additional possibilities are heating by an X-ray halo, an AGN, shocks or post-AGB stars. NGC 2768 lacks an X-ray halo (Komossa et al. 1999), so this option is ruled out. The $\mathrm{H} \beta$ flux in NGC 2768 does not drop off with the square of the radius as it should if ionised solely by an AGN (Hatch, Crawford \& Fabian 2007), therefore everything outside the central $2^{\prime \prime}$ must be ionised by another source (Sarzi et al., in preparation).

The most prominent feature of the ionised gas in NGC 2768 is a roughly spiral-shaped region of increased [O III] equivalent width (see fig. 4b, Sarzi et al. 2006). This strong enhancement in [OIII] without a corresponding strong enhancement in $\mathrm{H} \beta$ is seen in other SAURON galaxies (NGC 2974, NGC 3414) and must be caused by shock ionisation of the gas, as post-AGB stars are too symmetric to cause such asymmetric ionisation and star formation would not cause such an increase in [OIII]. Yet the smooth and circular $\mathrm{H} \beta$ emission suggests that an ionisation source other than shocks is responsible for the ionisation outside of the spiral pattern. Both star formation and post-AGB stars remain options. Post-AGB stars must be present in such an old galaxy and thus must play a role. However, the $\mathrm{H} \beta$ equivalent width map from SAURON shows a weak enhancement along the extended molecular gas seen to the northeast of the galaxy centre (see Fig. 17), pointing to star formation in this ionised gas.
The FIR luminosity ( $\left.\mathrm{L}_{\mathrm{FIR}}\right)$ to molecular mass $\left(\mathrm{M}_{\mathrm{H}_{2}}\right)$ ratio also provides possible evidence of star formation (Combes et al. 2007). We computed a bolometric FIR flux for NGC 2768,

$$
F I R \equiv 1.26 \times 10^{-14} \mathrm{~W} \mathrm{~m}^{-2}\left(2.58 S_{60 \mu \mathrm{m}}+S_{100 \mu \mathrm{m}}\right),
$$

where $S_{60 \mu \mathrm{m}}$ and $S_{100 \mu \mathrm{m}}$ are IRAS fluxes in Jy. The total FIR luminosity is then $\mathrm{L}_{\mathrm{F} I R}=($ FIR $) 4 \pi D^{2}=4.3 \times 10^{8}$ $\mathrm{L}_{\odot}$, giving a $\mathrm{L}_{\mathrm{FIR}}$ to $\mathrm{M}_{\mathrm{H}_{2}}$ ratio of $6.3 \mathrm{~L}_{\odot} / \mathrm{M}_{\odot}$, a reasonable value for a star-forming spiral (Devereux \& Hameed 1997). In spiral galaxies, FIR emission traces star formation activity and the ratio of $\mathrm{L}_{\mathrm{FIR}}$ to $\mathrm{M}_{\mathrm{H}_{2}}$ is assumed to measure how efficiently stars are being formed out of the molecular hydrogen. If star formation is the dominant process contributing to FIR emission in NGC 2768, then star formation is proceeding at a normal efficiency. However, other sources (such as an AGN) may significantly contribute to the FIR emission in early-type galaxies, weakening this conclusion. We can still use the FIR luminosity to give an upper limit to the star formation rate, deriving an upper limit of 0.07 solar masses per year Kennicutt 1998, eqn. 4). Thus if star formation is present in NGC 2768, it is occurring at a very low rate, and the timescale to exhaust the supply of molecular gas is around 1 Gyr.

As we have only unclear evidence of ongoing star formation in NGC 2768, we consider whether the molecular gas disc might be gravitationally stable and thus unable to form stars. Theoretical models from Kawata et al. (2007) suggest that circumnuclear discs in massive galaxies are more stable than those in less massive galaxies. A stabil- 
The molecular polar disc in NGC 2768

ity analysis of the molecular circumnuclear disc in 3C 31 based on Toomre's Q-parameter (Toomre 1964) suggests that it is gravitationally stable and thus unable to form stars (Okuda et al. 2005). Our data are insufficient to calculate Toomre's $Q$ parameter by themselves, but comparing with the models of Kawata et al. (2007) (see their fig. 5), we find that the $0.5 \mathrm{kpc}$ radius and $6.8 \times 10^{7} \mathrm{M}_{\odot}$ mass of the molecular disc in NGC 2768 should be unstable, with a $Q$ parameter less than 1 . Thus it is reasonable to expect star formation in the polar disc of NGC 2768.

\subsection{Origin of the $\mathrm{mm}$ continuum}

From radio and X-ray observations, we know that NGC 2768 is a low-luminosity AGN. Very Large Array (VLA) and Very Long Baseline Array (VLBA) observations indicate a compact radio source with no evidence of jets (Nagar et al. 2005). Most if not all X-ray emission is consistent with a point-source origin, and the X-ray count rate differed between observations a month apart, indicating that the X-ray source is variable (Komossa et al. 1999).

The few LLAGN that have been studied at millimetre frequencies show a range of spectral shapes attributed to varying contributions from optically thin synchrotron emission, self-absorbed synchrotron emission, and free-free emission (see Nagar et al. 2002; Doi et al. 2005; Krips et al. 2007). In Fig. 8, we plot the radio through FIR SED of NGC 2768, which is similar to those of some of the other LLAGN previously observed. Values and references are listed in Table 4. We note that the flux densities at different frequencies are necessarily at different resolutions and thus are not entirely comparable. However, as no extended structures are observed at the milliarcsecond resolution of the VLBA at $5 \mathrm{GHz}$, and the $1.4,15$, and $115 \mathrm{GHz}$ detections are all consistent with a point-source origin, we assume all these flux densities are dominated by an unresolved source and can thus be compared without correction. The upperlimit at $230 \mathrm{GHz}$ is also for a point source at the phase centre at the resolution of $1^{\prime \prime} 2 \times 1^{\prime \prime} .2$, while the $240 \mathrm{GHz}$ detection is a bolometric measurement from an $11^{\prime \prime}$ beam, which could include a contribution from cold dust (Wiklind \& Henkel 1995) as well as from a central AGN. These two observations are thus not as contradictory as they appear in the SED plot. However, we note that our map of $1 \mathrm{~mm}$ continuum emission does not trace the dust pattern seen in Fig. 6 nor do we see evidence of more emission in the central $11^{\prime \prime}$ after smoothing our data to this resolution.

First, the lack of strong, coincident $1 \mathrm{~mm}$ continuum emission rules out cold dust as the origin of the $3 \mathrm{~mm}$ continuum emission, as the thermal spectrum from dust drops sharply towards $\mathrm{mm}$ wavelengths (see the dust curves in Fig. 8). We can also rule out free-free emission using the formula in Doi et al. (2005) which assumes an electron temperature of $10^{4} \mathrm{~K}$ and $\mathrm{N}\left(\mathrm{He}^{+}\right) / \mathrm{N}\left(\mathrm{H}^{+}\right)=0.08$ :

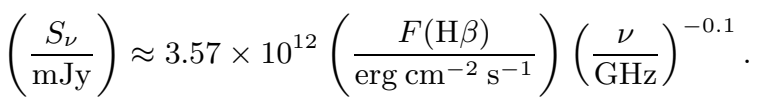

We use the total flux in $\mathrm{H} \beta$ from the SAURON data (Sarzi et al. 2006), which gives an overestimate for the central radio emission, as most of the $\mathrm{H} \beta$ emission comes from ionising sources other than star formation and only the flux
Table 4. Spectral energy distribution of NGC 2768.

\begin{tabular}{lccc}
\hline Instrument & Frequency $(\mathrm{GHz})$ & Flux Density $(\mathrm{mJy})$ & Ref. \\
\hline WRST & 1.4 & 10.9 & 1 \\
VLBA & 5 & 7.3 & 2 \\
VLA & 15 & 8.2 & 2 \\
PdB & 115 & 1.94 & 3 \\
PdB & 230 & $<2.25$ & 3 \\
IRAM 30m & 240 & 10.1 & 4 \\
MIPS & 1875 & 377 & 5 \\
IRAS & 3000 & 1370 & 6 \\
MIPS & 4286 & 694 & 5 \\
IRAS & 5000 & 390 & 6 \\
\hline
\end{tabular}

References - (1) Morganti et al. (2006); (2) Nagar et al. (2005); (3) this paper; (4) Wiklind \& Henkel (1995); (5) Temi et al. (2007); (6) Moshir et al. (1990).

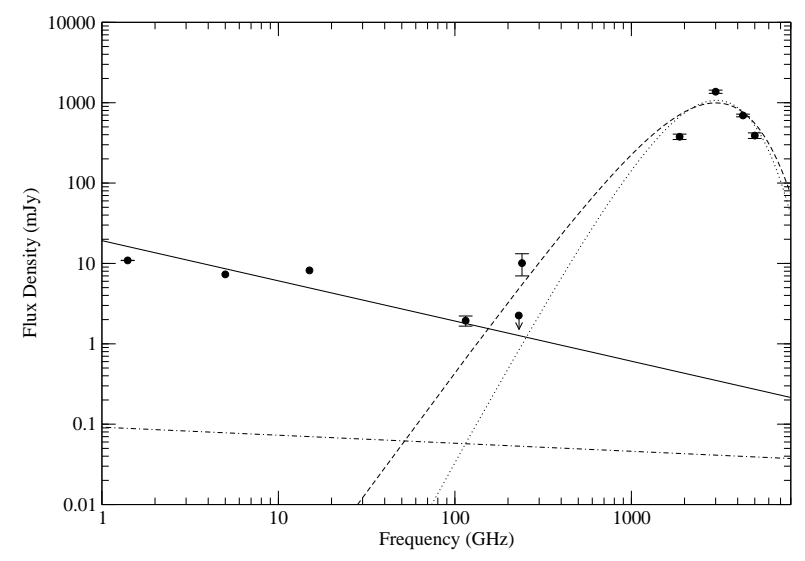

Figure 8. Spectral energy distribution of NGC 2768 from the radio to the far infrared (FIR). The solid line is an $\alpha=-0.5$ power law fit, modelling synchrotron emission. The dot-dashed line estimates the maximum contribution from free-free emission. The dashed curve extrapolates from the FIR dust bump to mm wavelengths using a $\beta=1$ emissivity law; the dotted curves does the same with $\beta=2$.

in the central pixels should correspond to $\mathrm{H}$ II regions that could appear as a central radio point source. Nevertheless, Fig. 8 reveals that, even with this overestimate, free-free emission is far too faint to account for the $3 \mathrm{~mm}$ continuum.

Having ruled out dust and free-free emission, processes directly related with an accreting supermassive black hole are likely to be the source of the $3 \mathrm{~mm}$ continuum detected in NGC 2768. Optically thin synchrotron emission from compact jets or other ejecta will produce a spectrum with a spectral index of $\alpha \leqslant-0.5$. The logarithmic least-squares fit of an $\alpha=-0.5$ power law is shown in Fig. 8 It roughly fits the 3 radio data points and the $3 \mathrm{~mm}$ continuum emission, but the low value at $1.4 \mathrm{GHz}$ and the high value at $15 \mathrm{GHz}$ hint at a flatter or inverted spectrum, suggesting synchrotron self-absorption plays a role. 


\section{CONCLUSIONS}

The interferometric observations of the $\mathrm{CO}(1-0)$ and $\mathrm{CO}(2-$ 1) lines presented here reveal a kiloparsec polar ring or disc of molecular gas within the early-type galaxy NGC 2768 . This molecular gas reveals the potential of this galaxy to form kinematic substructure through star formation. However, neither the kinematics nor the linestrengths of NGC 2768 as measured by SAURON show clear evidence of a young stellar population. This limits any star formation coincident with the molecular gas to be very recent and/or too small a luminosity-weighted fraction of the background old stellar population to contribute to the linestrengths and kinematics. The asymmetric and unrelaxed nature of the $\mathrm{CO}(1-0)$ emission suggests that the molecular gas in NGC 2768 was recently accreted and is possibly still being accreted, in agreement with the much more extended but disturbed H I distribution. Searching the neighbourhood of NGC 2768 for potential gas-donor galaxies, many H Irich low-luminosity galaxies are found, all of which could be the source of $\mathrm{H}$ I seen in and around NGC 2768, although UGC 4808 seems the most likely, being closest in projected distance and aligned with the extended H I distribution.

These CO observations of NGC 2768 probe new regions of parameter space in terms of molecular gas in early-type galaxies, with recently accreted, polar-rotating gas of only $6.8 \times 10^{7} \mathrm{M}_{\odot}$. As surveys have indicated lower CO detection rates in higher-luminosity E and S0 galaxies (Lees et al. 1991; Sage. Welch \& Young 2007), NGC 2768 is also notable for being the brightest in B-band of the $\mathrm{CO}$ detections in Combes et al. (2007). Whether the lack of observed star formation has to do with the (short) time since the gas has been accreted, the (low) mass of molecular gas available for star formation, the polar dynamics of the gas, or some host-galaxy property will take more investigation to discover, including an analysis of the molecular gas distributions of other early-type galaxies with integral-field data.

\section{ACKNOWLEDGEMENTS}

We would like to thank Philippe Salome for help with the reduction of the Plateau de Bure data and Marc Sarzi for useful discussions. We are also grateful to the SAURON Team for providing SAURON data as well as Tom Oosterloo for providing the H I datacube of NGC 2768. LMY acknowledges support from grant NSF AST-0507432 and would like to thank the Oxford Astrophysics Department for its hospitality during sabbatical work. We would also like to thank the anonymous referee for helpful comments which improved the paper.

The dust maps used observations made with the NASA/ESA Hubble Space Telescope, obtained from the data archive at the Space Telescope Institute. STScI is operated by the association of Universities for Research in Astronomy, Inc. under the NASA contract NAS 5-26555. The NASA/IPAC Extragalactic Database (NED) is operated by the Jet Propulsion Laboratory, California Institute of Technology, under contract with the National Aeronautics and Space Administration. This research made use of HyperLEDA: http://leda.univ-lyon1.fr. The Digitized Sky Surveys were produced at the Space Telescope Science Institute under U.S. Government grant NAG W-2166. The images of these surveys are based on photographic data obtained using the Oschin Schmidt Telescope on Palomar Mountain and the UK Schmidt Telescope.

\section{REFERENCES}

Bournaud F., Combes F., A\&A, 401, 817

Broeils A. H., van Woerden H., 1994, A\&AS, 107, 129

Combes F., Young L.M., Bureau M., 2007, MNRAS, 377, 1795

de Vaucouleurs G., de Vaucouleurs A., Corwin H. G., Buta R. J., Paturel G., Fouque P., 1991, Third Reference Catalog of Bright Galaxies, Vols. 1-3, XII. Springer-Verlag, Berlin (RC3)

de Zeeuw T., et al., 2002, MNRAS, 329, 513

Devereux N. A., Hameed S., 1997, ApJ, 113, 599

Djorgovski S., Davis M., 1987, ApJ, 31359

Doi A., Kameno S., Kohno K., Nakanishi K., Inoue M., 2005, MNRAS, 363, 692

Dressler A., Lynden-Bell D., Burstein D., Davies R. L., Faber S. M., Terlevich R., Wegner G., 1987, ApJ, 313, 42

Emsellem E., Greusard D., Combes F., Friedl D., Leon S.,

Pécontal E., Wozniak H., 2001, A\&A, 368, 52

Emsellem E., et al., 2004, MNRAS, 352, 721

Emsellem E., et al., 2007, MNRAS, 379, 401

Garcia A. M., 1993, A\&AS, 100, 47

Guilloteau S., Lucas R., 2000, in Mangum J.G., Radford S.J.E., eds, ASP Conf. Ser. Vol. 217, Imaging at Radio through Submillimeter Wavelengths. Astron. Soc. Pac., San Francisco, p. 299

Hatch N. A., Crawford C. S., Fabian A. C., 2007, MNRAS, 380, 33

Haynes M. P., Magri C., Giovanelli R., Starosta B. M., 1988, AJ, 95, 607

Heckman T. M., 1980, A\&A, 87, 152

Ho L. C., Filippenko A. V., Sargent W. L., 1997, ApJS, 98, 477

Högbom J. A., 1974, A\&AS, 15, 417

Jesseit R., Naab T., Peletier R. F., Burkert, A., 2007, MNRAS, 376, 997

Kawata D., Cen R., Ho L. C., 2007, ApJ, 669, 232

Kennicutt, R. C. Jr., 1998, ARA\&A, 36, 189

Kim D., ApJ, 346, 653

Knapp G.R., Guhathakurta P., Kim D., Jura M.A., 1989, ApJS, 70, 329

Komossa S., Böhringer H., Huchra J.P., A\&A, 349, 88

Krips M., et al., 2007, A\&A, 464, 553

Kuntschner H., et al., 2006, MNRAS, 369, 497

Lees J., Knapp G. R., Rupen M. P., Phillips T. G., 1991, ApJ, 379, 177

McDermid R.M., et al., 2006, MNRAS, 373, 906

Morganti R. et al., 2006, MNRAS, 371, 157

Moshir M. et al., 1990, IRAS Faint Source Catalog, version 2.0

Nagar N.M., Falcke H., Wilson A.S., 2005, A\&A, 435, 521

Nagar N. M., Wilson A. S., Falcke H., Ulvestad J. S., Mundell C. G., 2002, ASPC, 258, 171

Okuda T., Kohno K., Iguchi S., Nakanishi K., 2005, ApJ, 620,673

Sage L. J., Welch G. A., 2006, ApJ, 644, 850

Sage L. J., Welch G. A., Young, L. M., 2007, ApJ, 657, 232 
Sandage A., Bedke J., 1994, The Carnegie Atlas of Galaxies. DC: Carnegie Institute of Washington with The Flintridge Foundation, Washington DC (CAG)

Sarzi M. et al. 2006, MNRAS, 366, 1151

Sault R. J., Teuben P. J., Wright M. C. H., 1995, ASPC, 77,433

Schinnerer E., Scoville N., 2002, ApJ, 577, L103 2004, AJ, 127, 2641

Simien F., Prugniel P., 1997, A\&AS, 126, 519

Swaters R., 1999, PhD thesis, Rijksuniversiteit Groningen

Temi P., Brighenti F., Matthews W.G., 2007, ApJ, 660, 1215

Toomre A., 1964, SpJ, 139, 1217

Wozniak H., Combes F., Emsellem E., Friedli D., 2003, A\&A, 409, 469

Wiklind T., Henkel C., 1995, A\&A, 297, L71

Young L. M., 2002, ApJ, 124, 788

Young L. M., 2005, ApJ, 634, 258

Young L. M., Bureau M., Cappellari M., 2008, ApJ, accepted (astro-ph/0712.4189) 